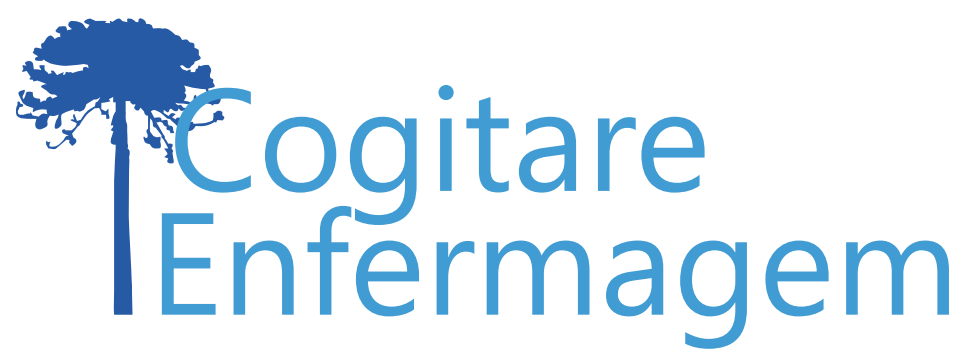

\title{
APRENDER PRÁTICA BASEADA NA EVIDÊNCIA PELO ENVOLVIMENTO EM ATIVIDADES DE INVESTIGAÇÃO - AUTOPERCEPÇÃO DOS ESTUDANTES
}

\author{
Mário Cardoso ${ }^{1}$ (1) \\ Cristina Lavareda Baixinho ${ }^{1}$ (i) \\ Óscar Ferreira ${ }^{1}$ (1) \\ Patricia Nascimento ${ }^{2}$ \\ Rita Pedrosa ${ }^{2}$ (1) \\ Paulo Gonçalves ${ }^{2}$ (1)
}

\begin{abstract}
RESUMO
Objetivo: compreender como os estudantes percepcionam o seu envolvimento em atividades de investigação e o contributo para o desenvolvimento de uma prática baseada na evidência.

Método: estudo qualitativo, transversal, descritivo e exploratório, realizado em Portugal, em agosto de 2019, que recorreu ao grupo focal, com oito participantes, para responder à questão: "Quais as vantagens da participação dos estudantes de enfermagem em atividades de investigação para o desenvolvimento de conhecimentos, atitudes e competências de utilização da evidência?". A análise de conteúdo foi realizada com o software NVivo.

Resultados: da análise qualitativa dos achados, emergiram cinco categorias e subcategorias: autoaprendizagem, integração teórico-prática, trabalho interdisciplinar, tomada de decisão baseada na evidência e literácia científica.

Conclusão: a criação de uma experiência eficaz de aprendizagem ajuda na construção do conhecimento e potencialmente contribui para a saúde da comunidade, os resultados de aprendizagem do estágio e o desenvolvimento de competências essenciais para uma Prática Baseada na Evidência.
\end{abstract}

DESCRITORES: Prática Clínica Baseada em Evidências; Aprendizagem; Estudantes; Enfermagem; Gestão do Conhecimento.

\section{APRENDIZAJE DE PRÁCTICAS BASADAS EN EVIDENCIA POR MEDIO DEL INVOLVIMIENTO EN ACTIVIDADES INVESTIGATIVAS - LA AUTOPERCEPCIÓN DE ESTUDIANTES}

\section{RESUMEN:}

Objetivo: comprender como estudiantes perciben su envolvimiento con actividades investigativas y su contribución para desarrollar una práctica basada en evidencias. Método: estudio cualitativo, trasversal, descriptivo y exploratorio, hecho en Portugal en agosto de 2019, utilizando un grupo focal con ocho participantes para responder a la cuestión: "Cuales las ventajas de la participación de los estudiantes de enfermería en actividades de investigación para desarrollar conocimientos, actitudes y competencias de utilización de la evidencia?" Se hizo al análisis de contenido con el software NVivo. Resultados: el análisis cuantitativo de los datos generó cinco categorías y subcategorías: autoaprendizaje, integración teórico-práctica, interdisciplinariedad, tomada de decisiones basadas en evidencia, y alfabetización científica. Conclusión: la creación de una experiencia eficaz de aprendizaje ayuda en la construcción del conocimiento y potencialmente contribuye para la salud de la comunidad, para los resultados del aprendizaje en la pasantía y para el desarrollo de competencias esenciales para una Práctica Basada en Evidencias.

DESCRIPTORES: Práctica Clínica Basada en Evidencias; Aprendizaje; Estudiantes; Enfermería; Gestión del Conocimiento. 
Os estudos sobre as vantagens de uma Prática Baseada na Evidência (PBE) na clínica têm possibilitado a emergência da discussão sobre as competências necessárias associadas à ciência, para que os futuros profissionais tenham conhecimentos sobre métodos e técnicas de investigação e utilizem a literácia científica como uma ferramenta de trabalho(1).

Este movimento tem influenciado os currículos dos cursos de enfermagem, nos quais, de um modo mais ou menos explícito, aparecem resultados de aprendizagem relacionados com a educação em PBE ${ }^{(1-2)}$. Todavia, alguns autores observam que a aprendizagem da PBE não se esgota no tradicional modelo de ensino escolar; implica a participação dos estudantes na investigação, possibilitando o desenvolvimento de capacidades de análise sobre o desenho do estudo, a sua qualidade e os resultados obtidos ${ }^{(2-4)}$.

É difícil aprender a pesquisar sem o envolvimento ativo dos estudantes, portanto, o professor não pode considerar que, ao preparar aulas (mesmo com métodos mais ativos de demonstração dos conteúdos), os alunos ficam aptos para utilizar a investigação e produzir resultados seguros na clínica(4).

Alguns pesquisadores, ao abordar a importância acadêmica da investigação e a sua relevância para a clínica, reforçam a importância da interligação dos cuidados prestados com a evidência científica ${ }^{(5-8)}$, e alertam que uma maior exploração de atividades de investigação pode capacitar os alunos de forma mais efetiva para a aquisição de competências fulcrais para a clínica( ${ }^{(5)}$, como a capacidade de pensamento crítico, pensamento analítico, resolução de problemas e raciocínio clínico(6).

Apesar do consenso sobre a relevância da evidência para a educação e profissão(6-7), constata-se que as disciplinas de investigação têm uma abordagem essencialmente teórica, que não possibilita, efetivamente, a competência no que toca à metodologia e condução de processos de investigação( ${ }^{(8)}$. Alguns autores fundamentam a ideia de que a PBE desenvolve-se pela participação dos futuros profissionais em projetos de pesquisa, aumentando a sua capacidade de leitura, produção e síntese de conhecimento. Por isso, as aulas teóricas de investigação devem ter associadas a outras estratégias que possibilitem a aquisição de habilidades e de atitudes científicas ${ }^{(1-4,6-7)}$.

Os Ensinos Clínicos (EC) podem desempenhar um papel importante na eliminação das dificuldades supracitadas, se possibilitarem a melhoria dos conhecimentos, atitudes e competências dos alunos ao nível da evidência científica ${ }^{(1-2,9)}$. A introdução da PBE o mais cedo possível nos currículos ${ }^{(8)}$, o investimento na participação em atividades de investigação ${ }^{(1,9)}$, a familiarização com o trabalho interprofissional a este nível, a interação efetiva com investigadores acadêmicos e clínicos e a incorporação dos resultados da investigação nas intervenções de Enfermagem ${ }^{(3)}$, interligados com os EC, podem proporcionar experiências enriquecedoras e com impacto futuro na utilização da $\mathrm{PBE}^{(1)}$.

Face ao exposto, é objetivo deste estudo compreender como os estudantes percepcionam o seu envolvimento em atividades de investigação e o contributo para o desenvolvimento de uma prática baseada na evidência.

\section{MÉTODO}

Estudo qualitativo, transversal, descritivo e exploratório, que recorreu ao grupo focal $(\mathrm{GF})^{(10-12)}$ para responder à questão: "Quais as vantagens da participação dos estudantes 
de enfermagem em atividades de investigação para o desenvolvimento de conhecimentos, atitudes e competências de utilização da evidência?".

A opção metodológica, inserida num paradigma interpretativo, justifica-se pela natureza do estudo, a especificidade do fenômeno em estudo e o estado da arte, dado que a revisão da literatura permitiu aferir que há poucos estudos sobre a participação dos alunos da formação pré-graduada em enfermagem em projetos de investigação(13).

O protocolo de pesquisa foi organizado em cinco etapas: planejamento; preparação; moderação; análise dos dados e divulgação dos resultados ${ }^{(12)}$.

A amostra intencional foi constituída por oito estudantes finalistas do Curso de Licenciatura em Enfermagem e segue as recomendações que defendem que o número ideal de participantes se situa entre cinco e dez participantes ${ }^{(11)}$. Os critérios de inclusão foram: ter realizado o ensino clinico de integração à vida profissional numa das intuições parceiras do Projeto Transição Segura, estar envolvido num dos projetos em curso nos diferentes serviços, não ter reprovado nesta unidade curricular em anos anteriores e manifestar interesse em participar.

Como reforçam os autores sobre a seleção dos constituintes dos GF, a amostra tinha uma característica em comum ${ }^{(10-12)}$ : a participação no projeto Transição Segura, no segundo semestre do ano letivo 2018/2019. Este é um projeto de Translação do Conhecimento para a clínica que envolve duas unidades de saúde e uma instituição de ensino superior e que, desde o seu início em 2015, envolve profissionais das áreas clínicas, acadêmicos e estudantes de enfermagem, com o intuito de promover uma Prática Baseada na Evidência(14). No decurso deste projeto, os alunos têm tido a possibilidade de integrar um conjunto de atividades de pesquisa e/ou translação do conhecimento para a praxis, que são creditadas como um complemento ao diploma destes futuros profissionais.

Apartir da questão central desta pesquisa, elaborou-se uma entrevista semiestruturada, com cinco perguntas norteadoras sobre as possibilidades que o envolvimento no projeto trouxe, ou não, em termos de trabalho interdisciplinar, pesquisa de evidência, utilização dos resultados de investigação nas suas intervenções, desenvolvimento de competências que envolvam pesquisa e transferência da evidência para os contextos de clínica.

O GF decorreu na primeira semana de agosto de 2019, após a saída da nota de EC, dado ser uma atividade extracurricular, que decorreu em simultâneo com o estágio do qual resultava uma nota final, com o intuito de diminuir/eliminar o efeito de respostas 'socialmente desejáveis'.

Os participantes foram previamente contactados, duas semanas antes do final do EC, por e-mail. Na informação enviada constava a data e hora, os objetivos, as regras de participação, incluindo tempo estimado de duração (75 minutos), com identificação do moderador e co-moderador da sessão(11-12). A opção por um co-moderador permitiu dividir papéis, ficando o moderador responsável pela condução e a manutenção da discussão e o co-moderador auxiliando a moderação, fazendo a gestão do equipamento de gravação, atento às condições logísticas e do ambiente físico e tomando notas sobre a discussão do grupo ${ }^{(11)}$.

Na semana, a entrevista e o papel do moderador e do co-moderador (observado) foi revisto pela equipe para garantir as competências de moderação, de dinâmica de grupo, de controle de possíveis elementos críticos para garantir o sucesso ${ }^{(12)}$.

O GF decorreu na escola de enfermagem e foi gravado em uma sala previamente preparada e o espaço organizado para promover um ambiente confortável, evitar interrupções e garantir o sigilo da informação(11-12). A gravação foi ouvida antes da transcrição. A transcrição foi efetuada por um dos investigadores presentes de forma a "visualizar" o que ocorreu no grupo; uma das tarefas mais intensas em termos de tempo deste método de coleta de informação é exatamente o processo de transcrição(12). 
A análise de conteúdo dos achados foi feita com o software NVivo. Num primeiro momento, foi feita a leitura flutuante da transcrição do GF, seguindo-se a codificação, com a seleção das unidades de codificação, a enumeração e a identificação das categorias. $O$ tratamento dos resultados possibilitou sua inferência e interpretação(11-12). Na definição das categorias, foi assegurada a representatividade, a exaustividade, a homogeneidade e a pertinência.

Este estudo foi aprovado por uma Comissão de Ética (Parecer 09/2019 HVFX). Foram respeitados todos os preceitos éticos e formais, desde a autorização institucional para os estudantes integrarem o projeto, enquanto atividade extracurricular, às questões éticas inerentes ao desenvolvimento da investigação. Foi assegurado a todos os participantes o anonimato e a confidencialidade dos dados e o direito à não participação.

\section{RESULTADOS}

Os participantes deste GF foram oito estudantes finalistas do curso de licenciatura em enfermagem, seis mulheres e dois homens, com média de idades de 22,3 1,98 anos.

Estes estudantes realizaram EC em diferentes serviços hospitalares: Unidade de Cuidados Intensivos (2), Serviço de Urgência (2), Ortopedia (2) e Medicina (2).

Da análise qualitativa dos achados, emergiram cinco categorias e respectivas subcategorias: Tomada de decisão baseada na evidência (Unidades de Registo (UR)=38); Literácia científica $(U R=33)$; Interdisciplinaridade $(U R=26)$; Integração teórico-prática $(U R=22)$ e Autoaprendizagem (UR=15) (Figura 1).
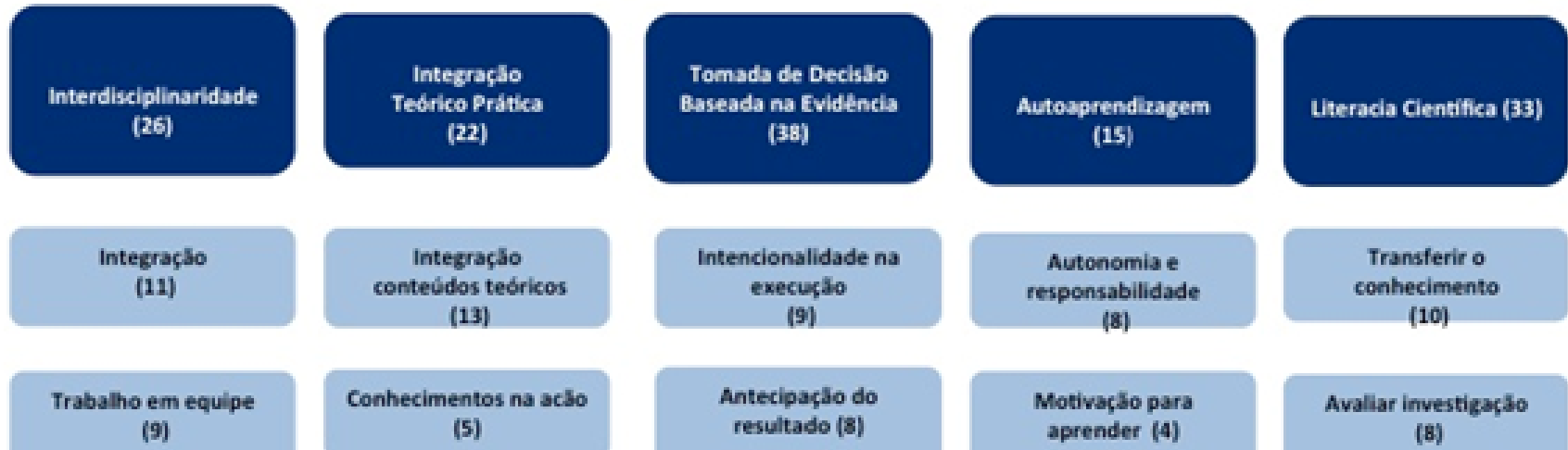

(9)

(5)
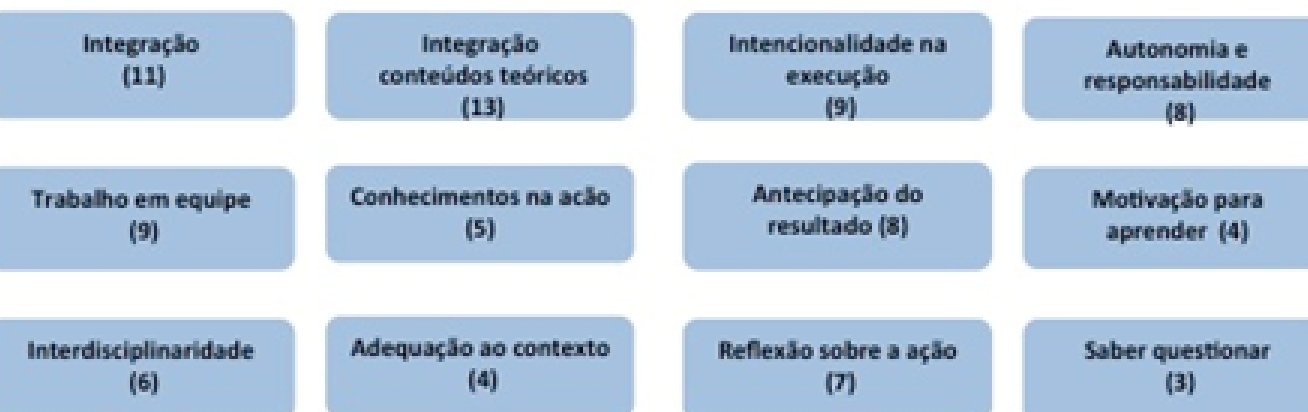

Interdisciplinaridade (6)

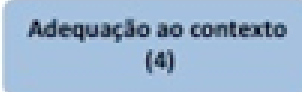

(4) aprender (4)

Saber questionar

(3)
Motivaç̧̆o para
(7)

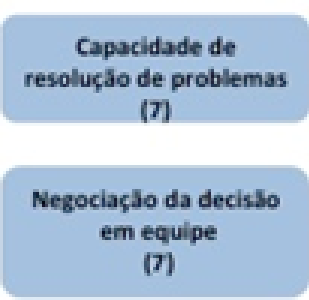

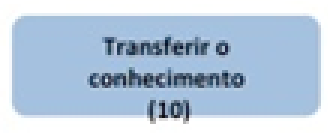

Avaliar investigaৎ̧ว

(8)

Interpretar resultados

(6)

Comunicar ciência (4)

Figura 1 - Categorias e subcategorias e respectivas Unidades de Registo. Lisboa, Portugal, 2020 Fonte: Autores (2020) 
A categoria que apresenta maior expressividade é a Tomada de decisão baseada na evidência, com 38 unidades de registo. Os participantes valorizam: a negociação da decisão em equipe, o tornar claro qual a intencionalidade por detrás da decisão, a antecipação do resultado, a reflexão sobre a ação e a capacidade de resolução de problemas.

O envolvimento dos participantes no projeto de translação de conhecimento era assegurado por um conjunto de atividades em que se incluía uma reunião antes do EC para negociação de objetivos e atividades (por ex. revisão da literatura com metodologia científica, coleta de dados, análise de dados, atividades e divulgação científica, criação de produtos para a transferência do conhecimento para clínica, entre outras). Este era o ponto de partida para a participação ativa do estudante, com supervisão nas atividades e participação nas reuniões de equipe onde, frequentemente, apareciam questões e dúvidas sobre como introduzir a evidência na clínica e que levava à reflexão sobre o processo para o fazer com segurança:

[...] vendo os aspectos positivos e negativos, decidindo qual a melhor forma de o fazer, prevendo resultados e havia sempre alguém do contra a perguntar os riscos (...) tínhamos que ver todos os ângulos. (E6)

A reflexão sobre a ação aumentava o questionamento sobre os modos de o fazer e as respectivas consequências das decisões, e implicava confrontar os modos de fazer, o saber dito teórico aprendido nas aulas de predomínio teórico e o que se observava no contexto.

[...] pensar que poderia ser diferente se fizer assim ou de outra forma. (E3)

A discussão e a negociação que envolviam as atividades extracurriculares foram transversais à própria obtenção dos objetivos do EC, o que aumentou a intencionalidade na execução das intervenções pelos estudantes. A integração possibilitou um maior sentimento de pertença ao serviço, proporcionando uma maior abertura para colocar questões, esclarecer dúvidas, possibilitando a integração de saberes e práticas cotidianas essenciais à tomada de decisão.

[...] o falarmos abertamente sobre as nossas dúvidas, incertezas, fez termos a certeza que os meios implementados eram os certos. Mas esta postura foi comum ao próprio estágio, dei por mim a colocar as minhas dúvidas sobre as técnicas e as intervenções sem medo, sem o receio de ser um estudante em avaliação, mas sim como alguém da equipe, que tal como os outros precisa discutir para decidir. (E2)

Associado à categoria Literácia científica $(U R=33)$, aparecem as subcategorias Transferir conhecimento; Avaliar investigação; Interpretar resultados e Comunicar ciência. Os participantes valorizam o aprender a transferir conhecimento de forma a permitir a integração de saberes e práticas cotidianas essenciais à construção de uma PBE:

[...] ajudou a pensar no processo de transferir o que aprendemos antes de ir para o estágio para a prestação de cuidados. (E7)

O desenvolvimento desta competência foi possível porque as atividades relacionadas com a avaliação e a interpretação dos resultados dos estudos permitiram fundamentar os projetos, inclusive a monografia de final de curso do estudante. Possibilitando introduzir na clínica intervenções, mais ou menos complexas, que induziram novos modos de fazer ou produziram mudanças nos atuais modos de fazer:

[...] O nosso projeto implicou a criação e uma consulta para seguimento dos doentes internados em cuidados intensivos, mesmo após a alta clínica. Fizemos pesquisa e identificamos os resultados da avaliação de outras consultas, o que ajudou na definição do tipo de consulta, instrumentos usados e intervenções implementadas (...) a análise do tipo de estudos e sua qualidade foi um desafio, mas que fez sentido perceber o porquê. (E1)

Ainda nesta categoria é de referir que quatro participantes valorizaram a experiência 
de participação em jornadas internacionais com pôsteres para a divulgação do projeto.

A Interdisciplinaridade $(U R=26)$ é muito apreciada pelos participantes, que registram que o fato de, em alguns serviços, as equipes multidisciplinares estarem envolvidas no projeto, promove uma integração rápida e com maior compromisso dos profissionais em suportar não só a atividade extracurricular, mas também o concretizar dos resultados de aprendizagem previstos para o EC:

[...] Desde o início do estágio que começamos em paralelo com a transição segura (...) desde o início fui aceito como uma deles, não era a estudante, era um elemento da equipe com funções e trabalho igual... não havia diferenciação. (E4)

A integração rápida e a aceitação do aluno no projeto tem vantagens para o processo de ensino-aprendizagem:

[...] diminui a incerteza que acompanha todos os períodos de EC, pelo desconhecimento das equipes, das suas dinâmicas e das expectativas que existem para o nosso desempenho. (E7)

A interdisciplinaridade é um instrumento básico de trabalho, que possibilita a aprendizagem do trabalho em equipes com profissionais diferentes, níveis de interdependência complexos e com impacto nos resultados dos cuidados às pessoas.

O percurso iniciado com a integração nos serviços possibilita o trabalho interdisciplinar entre os estudantes e os profissionais para um fim comum e transversal, independentemente da categoria profissional:

[...] fiquei surpresa quando me disseram para sentar e começaram a falar comigo de igual para igual, queriam saber a minha perspectiva para a consulta médica e de enfermagem (...) o que disse foi levado em conta e senti que contribuí para a mesma. (E2)

O trabalho em equipe, a rápida integração e a atitude acolhedora dos profissionais frente aos estudantes aumenta-lhes o sentimento de pertença (o Eu na equipe), facilita as relações interpessoais e atenua as relações de poder, que se estabelecem intra e interequipes.

A análise dos achados permite a compreensão das atividades em curso e do processo de transferência de conhecimento, o que promove a Integração teórico-prática. Os projetos trazem inovação e mudanças das práticas, o que implica a justificação (o porquê?) das atividades, com necessidade de identificar o que se sabe sobre o assunto e como o introduzir na clínica (adequação ao contexto). Esta categoria desvela-se no discurso oral com a percepção que a experiência contribuiu para atenuar aquilo que muitas vezes se denomina como o hiato entre a teoria e a prática:

[...] a participação no projeto permitiu aferir que aquele conhecimento é pertinente e que pode ser aplicado. (E3)

Esta integração do conhecimento teórico na ação torna consciente a integração dos "conteúdos teóricos" nos contextos de clínica, amenizando a noção que teoria e prática são dois mundos diferentes, como observam os fragmentos do discurso de dois estudantes:

[...] ver como se pode aplicar na prática o que se aprende na escola. (E6)

[...] na escola aprendemos, mas nem sempre vemos exemplos práticos tão próximos da teoria. (E8)

A Categoria com menor expressividade $(U R=15)$ é a Autoaprendizagem e respectivas subcategorias: Saber questionar; Autonomia e responsabilidade e Motivação para aprender. O envolvimento nas atividades do projeto Transição Segura aumentou a responsabilidade sobre os resultados que tinham de obter, conferiu autonomia e motivou-os, como expresso 
nestes trechos:

[...] saber que há um projeto para o qual o nosso trabalho vai contribuir, mais à frente, motiva para aprender e fazer bem. (E2)

[...] no nosso caso particular, a nossa pesquisa [Revisão Integrativa da Literatura] deu resultados que permitiram implementar uma consulta de follow up na unidade [de cuidados intensivos] e futuramente vai levar a uma mudança dos cuidados no serviço (....) à medida que tomamos consciência da importância, começamos a trabalhar mais. (E5)

\section{DISCUSSÃO}

Os achados deste estudo sugerem ganhos na participação ativa dos estudantes em projeto de Translação do Conhecimento para a clínica, sobretudo ao nível da tomada de decisão baseada na evidência e da literácia científica. $O$ conhecimento em saúde e o aparecimento de múltiplos modelos para transformar a evidência em políticas e práticas, com o propósito de implementar melhorias sustentáveis ${ }^{(9,15)}$, tem traduções na qualidade de vida da população e na diminuição dos gastos com os recursos de saúde ${ }^{(1)}$.

Os resultados tendem a confirmar que, para além do aumento do conhecimento sobre investigação, os participantes desenvolvem competências importantes para uma PBE tais como a tomada de decisão baseada na evidência, o saber questionar, o saber transferir o conhecimento para a clínica, o avaliar investigação, o interpretar resultados e o comunicar ciência. Outros estudos subsidiam estes achados ao recomendarem o envolvimento como uma estratégia importante para a aquisição de conhecimento e desenvolvimento de competências para adesão a uma verdadeira PBE, enquanto futuros profissionais ${ }^{(15-17)}$.

A participação ativa contribui para atingir um nível elevado de aprendizagem. Face aos achados, corroboramos a opinião de outros autores sobre a necessidade de investir em ambientes de prática clínica de qualidade e melhorar a supervisão e a avaliação da prática do estudante ${ }^{(1,18)}$.

A aprendizagem em contexto de EC possibilita implementar intervenções que solucionam problemas, melhoram cuidados e reduzem o tempo de internamento, promovendo a articulação entre níveis de cuidados $^{(1)}$. Os contextos clínicos e as situações clínicas dos doentes estão a tornar-se mais complexos, exigindo um aumento de competências científicas ${ }^{(6)}$ não só para o aumento da Literácia científica dos profissionais, mas também para facilitar a integração teórico-prática e potencializar novas aprendizagens ${ }^{(1)}$.

Outrossim é que a criação de experiências eficazes de aprendizagem promove a aquisição do conhecimento de enfermagem, e tem também a potencialidade de contribuir para a saúde da comunidade ${ }^{(16,19)}$ e pode potenciar os resultados de aprendizagem do próprio $\mathrm{EC}^{(1)}$.

A análise dos nossos achados reforça os resultados de outros estudos que concluem que a integração dos estudantes em projetos de pesquisa aumenta os níveis de motivação(20) e desenvolve competências de trabalho interprofissional ${ }^{(3,13)}$. Os alunos sentem-se motivados a guiar a sua aprendizagem para dar resposta aos inputs do contexto e, apesar do acréscimo de trabalho, manifestam entusiasmo e motivação porque verificam a curto prazo o seu contributo nas mudanças instituídas nos serviços. Ẻ importante desenvolver um sentimento de entusiasmo e gosto em relação à investigação e à sua relevância cotidiana (21).

Esta pesquisa revela que a decisão sobre a ação na clínica torna-se mais refletida e discutida na equipe, implicando a leitura, análise e transferência do conhecimento obtido por diferentes métodos, e aumentando a segurança na transferência do conhecimento 
produzido para os cuidados de saúde. Outros estudos reforçam que este envolvimento tem ganhos em termos de conhecimento e motivação, até pela constatação que a concretização das atividades que lhe são solicitadas ajuda no progresso de investigação( ${ }^{(3,6,13,16)}$.

Salienta-se que sendo esta uma atividade extracurricular, defende-se que os professores devem garantir que as aprendizagens se convertam em prática baseada na evidência nos contextos de prática clínica, onde, inseridos numa rede de cooperação interprofissional, os cuidados de enfermagem se tornam cada vez mais complexos ${ }^{(13,21)}$.

A participação favoreceu a interdisciplinaridade e a integração com os participantes, manifestando o sentido de pertença e de bem-estar nos serviços. A participação em programa de PBE permite o conhecimento dos vários papéis e o desenvolvimento de capacidade para trabalhar em equipes interdisciplinares ${ }^{(3)}$. Aparentemente sem ligação direta à PBE, a questão da integração e da comunicação intra e inter equipes é de grande importância porque os estudantes têm, nestas atividades, a possibilidade de liderar e promover o trabalho em equipe ${ }^{(17)}$ e, concomitantemente, desenvolvem competências comunicacionais fundamentais para a prestação de cuidados em contextos de saúde; almejase que sejam capazes de as mobilizar no estabelecimento de uma relação terapêutica com o cliente ${ }^{(22)}$.

Os achados expõem a intenção em continuar a valorizar a PBE enquanto futuros profissionais. No entanto, não é possível prever esse impacto, porque o intervalo temporal entre a experiência tida e o GF é curta, o que pode influenciar a intenção. Este aspecto deve ser explorado em estudos futuros. Ressalva-se que este é um estudo exploratório, limitado a um contexto muito específico, mas cujos resultados contribuem para a discussão em torno da ideia que pesquisar durante a licenciatura é um foco emergente para ajudar os alunos a desenvolver as habilidades e competências necessárias para um profissional de saúde na atualidade ${ }^{(1,16)}$.

Algumas pesquisas constatam que os estudantes apresentam baixos níveis de conhecimento, atitude e intenção de implementar uma PBE ${ }^{(23)}$. Todavia, os nossos achados reforçam a importância da continuidade da participação dos mesmos neste tipo de projeto. A universidade tem um dever moral na preparação de licenciados competentes para fornecer uma base de qualidade e evidência nos cuidados de enfermagem ${ }^{(1)}$.

As limitações do estudo estão associadas ao método e ao contexto. A entrevista em grupo pode ter influenciado a interação e a resposta individual. A pesquisa avaliou uma experiência num contexto especifico e limitado, não permitindo generalizações. Porém, numa área em que a investigação ainda é escassa, a opção por um estudo de natureza qualitativa possibilitou explorar o fenômeno em estudo. Os achados e sua discussão orientam para pesquisas futuras.

\section{CONSIDERAÇÕES FINAIS}

A revisão da literatura possibilitou observar que existe pouca evidência sobre o envolvimento dos estudantes, da formação pré-graduada, em projetos de pesquisa. Os achados deste grupo focal reforçam a necessidade de se repensar o lugar da prática baseada na evidência nos currículos dos cursos de licenciatura em enfermagem, não só em termos de conteúdo teórico, mas também criando oportunidades de participação dos estudantes em atividades de investigação, promovendo a aquisição de conhecimentos, atitudes e competências.

Da análise do discurso dos oitos participantes neste estudo exploratório, inserido num paradigma qualitativo, emergiram cinco categorias e subcategorias sobre o envolvimento de alunos num projeto de translação do conhecimento: Tomada de decisão 
baseada na evidência; Literácia científica; Interdisciplinaridade; Integração teórico-prática; e Autoaprendizagem.

Esta pesquisa tem implicações para a prática. A orientação clínica dos estudantes numa postura mais colaborativa é um desafio para os tutores e traz benefícios para os equipamentos de saúde com a introdução da evidência nos contextos da práxis.

\section{REFERÊNCIAS}

1. Baixinho CL, Ferreira Ó, Cardoso M, Nascimento P, Pedrosa R, Gonçalves P. Autopercepção dos estudantes sobre a participação em atividades extracurriculares de transferência de conhecimento: o exemplo da Transição Segura. NTQR. [Internet]. 2020 [acesso em 30 nov 2020]; 2. Disponível em: https:// doi.org/10.36367/ntar.2.2020.588-601.

2. Mena-Tudela D, González-Chordá VM, Cervera-Gasch A, Maciá-Soler ML, Orts-Cortés MI. Effectiveness of an Evidence-Based Practice educational intervention with second-year nursing students. Rev Latino-Am Enfermagem. [Internet]. 2018. [acesso em 27 fev 2020] 26. Disponível em: https://dx.doi. org/10.1590/1518-8345.2502.3026.

3. Slattery MJ, Logan BL, Mudge B, Secore K, Reyn LJ von, Maue RA. An Undergraduate Research Fellowship Program to Prepare Nursing Students for Future Workforce Roles. J Prof Nurs. [Internet]. 2016 [acesso em 27 fev 2020]; 32(6). Disponível em: https://doi.org/10.1016/j.profnurs.2016.03.008.

4. Sun JH, Liu JE, Wu Y, Li SJ. The effects of the student-centered clinical nursing practice mode based on the action research for clinical practicum of undergraduate students in Beijing, China. Procedia Soc Behav Sci [Internet]. 2014 [acesso em 26 fev 2020]; 141. Disponível em: http://doi.org/10.1016/j. sbspro.2014.05.146.

5. Egilsdottir HÖ, Byermoen KR, Moen A, Eide $H$. Revitalizing physical assessment in undergraduate nursing education - what skills are important to learn, and how are these skills applied during clinical rotation? A cohort study. BMC Nurs. [Internet]. 2019 [acesso em 27 nov 2020]; 18(41). Disponível em: http://doi.org/10.1186/s12912-019-0364-9.

6. Einarsen KA, Giske T. Nursing students' longitudinal learning outcomes after participation in a research project in a hospital. IPDJ. [Internet]. 2019 [acesso em 27 fev 2020]; 9(1). Disponível em: https://doi. org/10.19043/ipdj.91.004.

7. Aglen B. Pedagogical strategies to teach bachelor students evidence-based practice: a systematic review. Nurse Educ Today. [Internet]. 2016 [acesso em 30 nov 2020]; 36. Disponível em: https://pubmed. ncbi.nlm.nih.gov/26375570/.

8. Vera F. Research skills in nursing undergraduate students: a case study at a Chilean private university. OSJ. [Internet]. 2020 [acesso em 26 fev 2020]; 5(3). Disponível em: http://doi.org/10.23954/osj.v5i3.2487.

9. Kitson A, Brook A, Harvey G, Jordan Z, Marshall R, O'Shea R, Wilson D. Using complexity and network concepts to inform healthcare knowledge translation. Int J Health Policy Manag. [Internet]. 2018 [acesso em 26 fev 2020]; 7(3). Disponível em: http://doi.org/10.15171/IJHPM.2017.79.

10. Flynn R, Albrecht L, Scott SD. Two Approaches to Focus Group Data Collection for Qualitative Health Research: Maximizing Resources and Data Quality. Int J Qual Methods [Internet]. 2018 [acesso em 01 dez 2020]; 17. Disponível em: https://doi.org/10.1177/1609406917750781.

11. Krueger RA, Casey MA. Focus groups: a practical guide for applied research. 5 ed. New Delhi: Sage; 2014.

12. Silva IS, Veloso AL, Keating JB. Focus group: considerações teóricas e metodológicas. Rev Lusofona 
Educ. [Internet]. 2014 [acesso em 27 fev 2020]; 26(26). Disponível em: https://revistas.ulusofona.pt/index. php/rleducacao/article/view/4703.

13. Loura D, Bernardes R, Baixinho CL, Rafael H, Félix I, Guerreiro M. Aprender em projetos de investigação durante a licenciatura em enfermagem: revisão integrativa da literatura. NTQR. [Internet]. 2020 [acesso em 02 dez 2020]; 3. Disponível em: http://doi.org/10.36367/ntqr.3.2020.293-304.

14. Baixinho CL, Ferreira Ó, Marques FM, Presado MH, Cardoso M. Transição segura: um projeto da transferência do conhecimento para a prática clínica. In: Costa AP, Sánches-Gómez MC, Cilleros MVM. A prática na investigação qualitativa: exemplos de estudos. Oliveira de Azeméis: Ludomédia; 2017. p. 5780.

15. Baixinho $C L$, Costa AP. Do hiato no discurso teoria - prática à clinica baseada na unicidade do conhecimento. Esc. Anna Nery [Internet]. 2019 [acesso em 02 dez 2020]; 23(3). Disponível em: https://doi. org/10.1590/2177-9465-ean-2019-0141.

16. Long A, Bischoff WR, Aduddell K. Research prescription for undergraduate students: research mentoring in a small liberal arts university. J Prof Nurs [Internet]. 2019 [acesso em 27 fev 2020]; 35(3). Disponível em: https://doi.org/10.1016/j.profnurs.2018.12.007.

17. Jansen DA, Jadack RA, Ayoola AB, Doornbos MM, Dunn SL, Moch SD, et al. Embedding research in undergraduate learning opportunities. West J Nurs Res. [Internet]. 2015 [acesso em 28 fev 2020]; 37(10). Disponível em: http://doi.org/10.1177/0193945915571136.

18. Heidari MR, Norouzadeh R. Nursing students' perspectives on clinical education. J Adv Med Educ Prof. [Internet]. 2015 [acesso em 26 fev 2020]; 3(1). Disponível em: https://www.ncbi.n/m.nih.gov/pmc/ articles/PMC4291507/.

19. Jong G de, Meijer E, Schout G, Abma T. Involving undergraduate nursing students in participatory health Research: Implications from the Netherlands. J Prof Nurs [Internet]. 2018 [acesso em 27 fev 2020]; 34(6). Disponível em: https://doi.org/10.1016/j.profnurs.2018.04.001.

20. Kurtz CP, Kessler TA. An undergraduate collaborative team model to engage nursing students in research. J Nurs Educ Pract. [Internet]. 2017 [acesso em 30 nov 2020]; 7(7). Disponível em: https://doi. org/10.5430/jnep.v7n7p112.

21. Kim JS, Gu MO, Chang $\mathrm{H}$. Effects of an evidence-based practice education program using multifaceted interventions: a quasi-experimental study with undergraduate nursing students. BMC Med Educ. [Internet]. 2019 [acesso em 01 mar 2020]; 19(71). Disponível em: https://doi.org/10.1186/s12909019-1501-6.

22.Graan AC van, Williams MJS, Koen MP. Professional nurses' understanding of clinical judgement: a contextual inquiry. J Interdisc Health Sci. [Internet]. 2016. [acesso em 27 mar 2020]; 21. Disponível em: https://doi.org/10.1016/j.hsag.2016.04.001.

23. Ashktorab T, Pashaeypoor S, Rassouli M, Alavi-Majd H. Nursing students' competencies in evidencebased practice and its related factors. Nurs Midwifery Stud. [Internet]. 2015 [acesso em 27 mar 2020]; 4(4). Disponível em: https://www.ncbi.nlm.nih.gov/pmc/articles/PMC4733497/. 


\section{COMO REFERENCIAR ESTE ARTIGO:}

Cardoso M, Baixinho CL, Ferreira O, Nascimento P, Pedrosa $R$, Gonçalves P. Aprender prática baseada na evidência pelo envolvimento em atividades de investigação - autopercepção dos estudantes. Cogit. Enferm. [Internet]. 2021 [acesso em "colocar data de acesso, dia, mês abreviado e ano"]; 26. Disponível em: http://dx.doi. org/10.5380/ce.v26i0.79806.

Recebido em: 04/03/2021

Aprovado em: 15/03/2021

Editora associada: Luciana Puchalski Kalinke

Autor Correspondente:

Cristina Lavareda Baixino

Escola Superior de Enfermagem de Lisboa - Lisboa, Portugal

E-mail: crbaixinho@esel.pt

Contribuição dos autores:

Contribuições substanciais para a concepção ou desenho do estudo; ou a aquisição, análise ou interpretação de dados do estudo - MC, CLB, OF, PN, RP

Elaboração e revisão crítica do conteúdo intelectual do estudo - MC, CLB, OF, PN, RP

Aprovação da versão final do estudo a ser publicado - MC, CLB, OF, PN, RP

Responsável por todos os aspectos do estudo, assegurando as questões de precisão ou integridade de qualquer parte do estudo - PG

ISSN 2176-9133

(c) (i)

Copyright (c) 2021 Este é um artigo em acesso aberto distribuído nos termos da Licença Creative Commons Atribuição, que permite o uso irrestrito, a distribuição e reprodução em qualquer meio desde que o artigo original seja devidamente citado. 